\title{
UNSTEADY CONVECTIVE HEAT AND MASS TRANSFER FLOW IN A THIN LIQUID FILM OVER MOVING SHEET IN A SATURATED PERMEABLE SURFACE
}

\author{
Md. Hasanuzzaman*, Romana Afroj and M.M. Touhid Hossain \\ Department of Mathematics, Khulna University of Engineering \& Technology, Bangladesh
}

Received: 17 April 2021

Accepted: 15 May 2021

\begin{abstract}
In this paper, the unsteady convective heat and mass transfer flow in a thin liquid film over moving sheet in a saturated permeable surface has been investigated. The sets of nonlinear couple partial differential equations involving the continuity, momentum, energy and mass balance equations have been reduced to two sets of nonlinear ordinary differential equations by using well know similarity technique. The transformed ordinary differential equations with associate boundary conditions are then solved numerically by using shooting technique with the help of 'ODE45 MATLAB' software. The numerical solution regarding the non-dimensional temperature and concentration variables against the non-dimensional coordinate variable have been carried out for different values of pertinent parameters and numbers like Prandlt number $\left(P_{r}\right)$, Darcy number $(D a)$, Schmidt number $(S c)$ and other driving parameters. The numerical results obtained of the skin friction coefficient, the local Nusselt number and the local Sherwood number have been presented graphically and also in tabular form. The result indicates that the velocity decrease with increasing values of Darcy number but opposite behavior for the temperature and the concentration. The heat transfer rate increases due to the increase in magnitude of Prandtl number so that the temperature reduces and the higher values of Schmidt number representing heavier species, increase of Schmidt number lower down the concentration level in the boundary layer.
\end{abstract}

Keywords: Heat and mass transfer, moving sheet, permeable surface, thin liquid film.

\section{INTRODUCTION}

In recent years, a great attention has been focused the researches of fluid flow and convective heat transfer along a vertical flat plate embedded in a permeable surface because of its vase-range of application in different areas such as geophysical flow, water pollution of transfer ground, thermal insulation, processes of oil recovery, processing of food manufacture and the enhanced recovery of petroleum resource, etc. In permeable medium, the sea bed and disposal of nuclear wastage into the earth's crust in the research of convection is an area of particular interest. The fluid dynamics researchers have been received a great attention to interpret and explain how these non-Newtonian fluids flow along vertical and horizontal stretching surface Huang et al. (1989) and Rashad et al. (2011). There are many outstanding applications with yield stress on convective heat transport of non-Newtonian power-law in fluid-saturated permeable surfaces considering oil reservoir and geothermal engineering application presented be Shenay (1993). Nield and Bejan (1984) explained Darcy and non-Darcy mixed convection researches in detailed. Chen and Chen (1988) have been explained that in a non-Newtonian fluid natural convection about a sphere and horizontal sphere along a vertical plate embedded in a permeable surface. In permeable medium, the combined forced and free convection flow about in the inclined surface has been investigated by Cheng (1977). Also, in permeable surface, for the case of opposing flow with constant temperature dual solutions occurring in the problem of the mixed convection flow over a vertical plate which has been studied by Merkin (1985). Mixed convection from a vertical plate in permeable surface with suction and blowing have been explained by Hooper et al. (1993). From a vertical plate the free convection with a predefined surface heat flux in a permeable medium have been studied by Wright et al. (1996). The natural convection about a cone embedded in a saturated permeable medium with uniform lateral mass flux has been numerically examined by Yih (1997).

The free convection flow phenomena of several non-Newtonian fluids have been considered in Acrivos (1960) and Shenoy (1994). Using the flow model of a non-Newtonian power law fluid Emery et al. (1970) and Chen and Wollersheim (1973) etc. studied the problems of free convection in order to predict the characteristics of steady natural convection flow and heat transfer along an isothermal vertical plate embedded in a permeable surface. Natural convection flow of non-Newtonian fluids along a vertical wavy surface with uniform surface temperature as well as uniform heat flux has been considered by among others Molla and Yao (2009) and Degan et al. (2007).

An analytical approach based on modified Darcy power-law model has been carried out by Degan et al. (2007) in order to investigate the transient natural convection boundary layer flow about a vertical surface embedded in 
an anisotropic permeable surface saturated by a non-Newtonian fluid. The problems of mixed convection flow along vertical surface for non-Newtonian fluid-saturated porous medium are investigated by Rashad et al. (2013). Khader and Megahed (2013) introduced a numerical simulation for the effect of thermal radiation on flow and heat transfer over an unsteady stretching sheet embedded in a permeable medium using finite difference method.

From the above research works, the present study will carry out the numerical investigation of unsteady convective heat and mass transfer in a thin liquid film over moving sheet in a saturated permeable surface. Computations have been performed for a wide range of the non-dimensional parameters such as Darcy number, Prandtl number, Schmidt number and other driving parameter on velocity, temperature and concentration fields. Also, numerical computations have been made for the local skin friction, local Nusselt number and the local Sherwood numbers for different values of pertinent numbers/parameters and are tabulated and graphically.

\section{GOVERNING EQUATIONS}

Physical model of the problem representing an elastic sheet issued from a narrow slot at the origin of a Cartesian coordinate system is shown in figure 1 . The liquid of interest is considered to be Newtonian fluid. The continuous surface aligned with the $x$-axis at $y=0$ moves in its own plane with a velocity $U(x, t)$. The temperature and concentration distributions at the surface are $T_{S}(x, t)$ and $C_{S}(x, t)$, respectively.

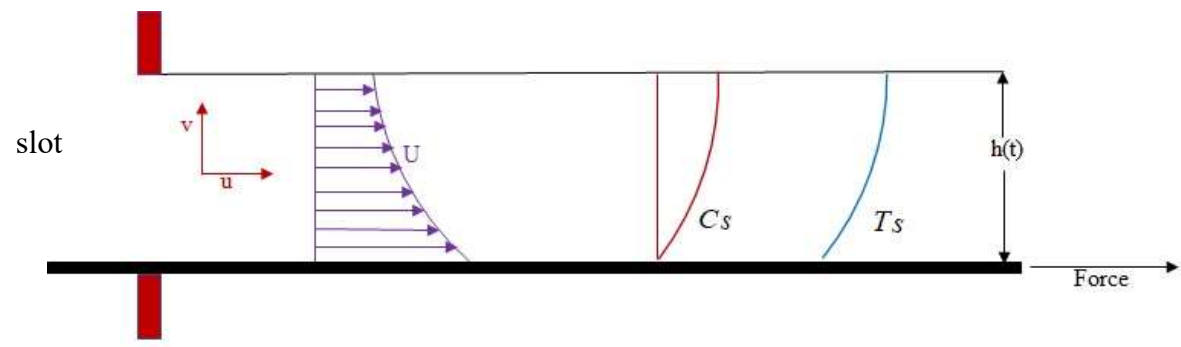

Figure 1: Physical model

The associate boundary layer equations for mass, momentum, energy conservation and mass balance governing the problem are in the following forms:

$$
\begin{aligned}
& \frac{\partial u}{\partial x}+\frac{\partial v}{\partial y}=0 \\
& \frac{\partial u}{\partial t}+u \frac{\partial u}{\partial x}+v \frac{\partial u}{\partial y}=\frac{\mu}{\rho} \frac{\partial^{2} u}{\partial y^{2}}-\frac{\mu}{\rho K} u \\
& \frac{\partial T}{\partial t}+u \frac{\partial T}{\partial x}+v \frac{\partial T}{\partial y}=\alpha \frac{\partial^{2} T}{\partial y^{2}} \\
& \frac{\partial C}{\partial t}+u \frac{\partial C}{\partial x}+v \frac{\partial C}{\partial y}=D^{*} \frac{\partial^{2} C}{\partial y^{2}}
\end{aligned}
$$

where $u$ and $v$ are the components of velocity along the $x$-axis and $y$-axis, respectively. $T$ is the fluid temperature, $C$ is the fluid concentration $t$ is the time, $\mu$ is the fluid viscosity, $K$ is the permeability of surface, $\rho$ is the fluid density, $\alpha$ is the thermal diffusivity and the $D^{*}$ is the coefficient of mass diffusion.

The above equations are subject the following boundary conditions:

$$
\begin{aligned}
& u=U, v=0, T=T_{S}, C=C_{S} \text { at } y=0 \\
& \frac{\partial u}{\partial y}=\frac{\partial T}{\partial y}=\frac{\partial C}{\partial y}=0 \text { at } y=h \\
& v=\frac{d h}{d t} \quad \text { at } y=h
\end{aligned}
$$

where $h$ is the liquid film thickness and $U$ is the surface velocity at $y=0$ and along $x$-direction the sheet continuously moves with the velocity

$$
U=\frac{b x}{1-a t}
$$

Here the positive constants are $a$ and $b$ with having dimension (time) $)^{-1}$. The surface temperature is $T_{S}$ and the surface concentration is $C_{S}$ of the moving sheet varies with the distance $x$ and time $t$ in the form:

$$
\begin{aligned}
& T_{S}=T_{0}-T_{\text {ref }}\left(\frac{b \rho^{2}}{2 \mu}\right)(1-a t)^{-\frac{3}{2}} \\
& C_{S}=C_{0}-C_{\text {ref }}\left(\frac{b \rho x^{2}}{2 \mu}\right)(1-a t)^{-\frac{3}{2}}
\end{aligned}
$$

where for all $t<\frac{1}{a}$, the temperature and concentration at the slit are $T_{0}$ and $C_{0}$, respectively and the constant reference temperature and concentration are $T_{\text {ref }}$ and $C_{\text {ref }}$, respectively.

Upon introducing the following similarity transformations: 


$$
\begin{aligned}
& \eta=\left(\frac{b \rho}{\mu}\right)^{\frac{1}{2}}(1-a t)^{-\frac{1}{2}} \beta^{-1} y \\
& u=b x(1-a t)^{-1} f_{\eta}(\eta) \\
& v=-\left(\frac{b \mu}{\rho}\right)^{\frac{1}{2}}(1-a t)^{-\frac{1}{2}} \beta f(\eta) \\
& T=T_{0}-T_{r e f}\left(\frac{b \rho x^{2}}{2 \mu}\right)(1-a t)^{-\frac{3}{2}} \theta(\eta) \\
& C=C_{0}-C_{r e f}\left(\frac{b \rho x^{2}}{2 \mu}\right)(1-a t)^{-\frac{3}{2}} \phi(\eta)
\end{aligned}
$$

Here Noor and Hashim (2010) are explained the non-dimensional thin film thickness $\beta$ and is given by

$$
\beta=\left(\frac{b \rho}{\mu}\right)^{\frac{1}{2}}(1-a t)^{-\frac{1}{2}} h(t)
$$

From the above transformations, the non-dimensional, nonlinear and couple ordinary differential equations have been obtained as follows:

$$
\begin{aligned}
& f_{\eta \eta \eta}(\eta)+\gamma\left[f(\eta) f_{\eta \eta}(\eta)-\frac{s}{2} \eta f_{\eta \eta}(\eta)-f_{\eta}^{2}(\eta)-(S+D) f_{\eta}(\eta)\right]=0 \\
& \frac{1}{P_{r}} \theta_{\eta \eta}(\eta)+\gamma\left[f(\eta) \theta_{\eta}(\eta)-2 f_{\eta}(\eta) \theta(\eta)-\frac{s}{2} \eta \theta_{\eta}(\eta)-\frac{3}{2} S \theta(\eta)\right]=0 \\
& \frac{1}{S c} \phi_{\eta \eta}(\eta)+\gamma\left[f(\eta) \phi_{\eta}(\eta)-2 f_{\eta}(\eta) \phi(\eta)-\frac{s}{2} \eta \phi_{\eta}(\eta)-\frac{3}{2} S \phi(\eta)\right]=0
\end{aligned}
$$

The dimensionless boundary conditions which are given below:

$$
\begin{aligned}
& f(0)=0, f_{\eta}(0)=1, \theta(0)=1, \phi(0)= \\
& f_{\eta \eta}(1)=0, \theta_{\eta}(1)=0, \phi_{\eta}(1)=0, f(1) \frac{s}{2}
\end{aligned}
$$

where $f_{\eta}(\eta), \theta(\eta), \phi(\eta)$ are the dimensionless velocity, temperature and concentration, respectively, the similarity variable is $\eta$, the unsteadiness parameter is $S=\frac{a}{b}$, the Prandtl number is $P_{r}=\frac{v}{\alpha}$, the dimensionless film thickness is $\gamma=\beta^{2}$, the Darcy number is $D=\frac{\mu(1-a t)}{\rho b K}$ and the Schmidt number is $S c=\frac{\mu}{\rho D^{*}}$.

The physical quantities of engineering interest in the present study are the local skin-friction coefficient $C_{f}$, the local Nusselt number $N u_{x}$ and the local Sherwood number $S h_{x}$, respectively have been given by:

$$
\begin{aligned}
& C_{f}=\frac{2}{\beta} f_{\eta \eta}(0) R e_{x}^{-\frac{1}{2}} \\
& N u_{x}=\frac{1}{2 \beta(1-a t)} \theta_{\eta}(0) R e_{x}^{\frac{3}{2}} \\
& S h_{x}=\frac{1}{2 \beta(1-a t)} \phi_{\eta}(0) R e_{x}^{\frac{3}{2}}
\end{aligned}
$$

where the local Reynolds number $R e_{x}=\frac{\rho U x}{\mu}$.

\section{NUMERICAL SOLUTIONS AND DISCUSSIONS}

An investigation for unsteady convective heat and mass transfer in a thin liquid film over moving sheet in a saturated permeable surface has been carried out to analyze the impacts of surface of permeability and thermal and mass diffusivity. The resulting initial value problems involving the set of ordinary differential equations (17) - (19) with the boundary conditions (20) - (21) are solved numerically by applying shooting technique through "ODE45 MATLAB" software. The effects of dimensionless film thickness $(\gamma)$, unsteadiness parameter $(S)$, Prandtl number $\left(P_{r}\right)$, Darcy number $(D a)$ and Schmidt number $(S c)$ are plotted in the figures 2 to 6 . To observe the effect of dimensionless film thickness $\gamma$, the rest parameters/numbers are treated as constants. Similarly, to investigate the influence of one parameter/number on the field variables, only the variations of that selected parameter/number has been made while the values of the other parameters/numbers are taken to be fixed. The constant values for different parameters/numbers are chosen $\gamma=16, D a=0.6, P_{r}=0.71, S c=0.5$, and $S=$ 0.8 .

Figure 2(a-c) represents the effect of dimensionless film thickness $\gamma$ on the velocity, temperature as well as concentration distributions, respectively. From Fig. 2(a) it is seen that for all cases the velocity is starting from 1(one), and then velocity decrease and finally asymptotically leads to zero with the increase of $\eta$. As seen in the Fig. 2(a) the velocity decrease with the increase of $\gamma$. From Fig. 2(b) and 2(c) it is clearly observed that with an increase in $\gamma$ at any point the temperature and concentration are reducing. Figures $3(\mathrm{a}-\mathrm{c})$ demonstrates the variation of the fluid velocity, temperature and concentration profiles for various values of Darcy parameter $D a$. It can be concluded from figure 3(a) that with an increase in Darcy number the velocity decreases along the sheet and the reverse is observed away from the sheet. It is revealed that the temperature and concentration at any point increase with increase in $D a$ are shown in Fig.3 (b) and 3(c). This is due to fact that the permeable surface creates a resistive type of force for which enhancing the heat and mass transfer and a reduction in the velocity of fluid. 

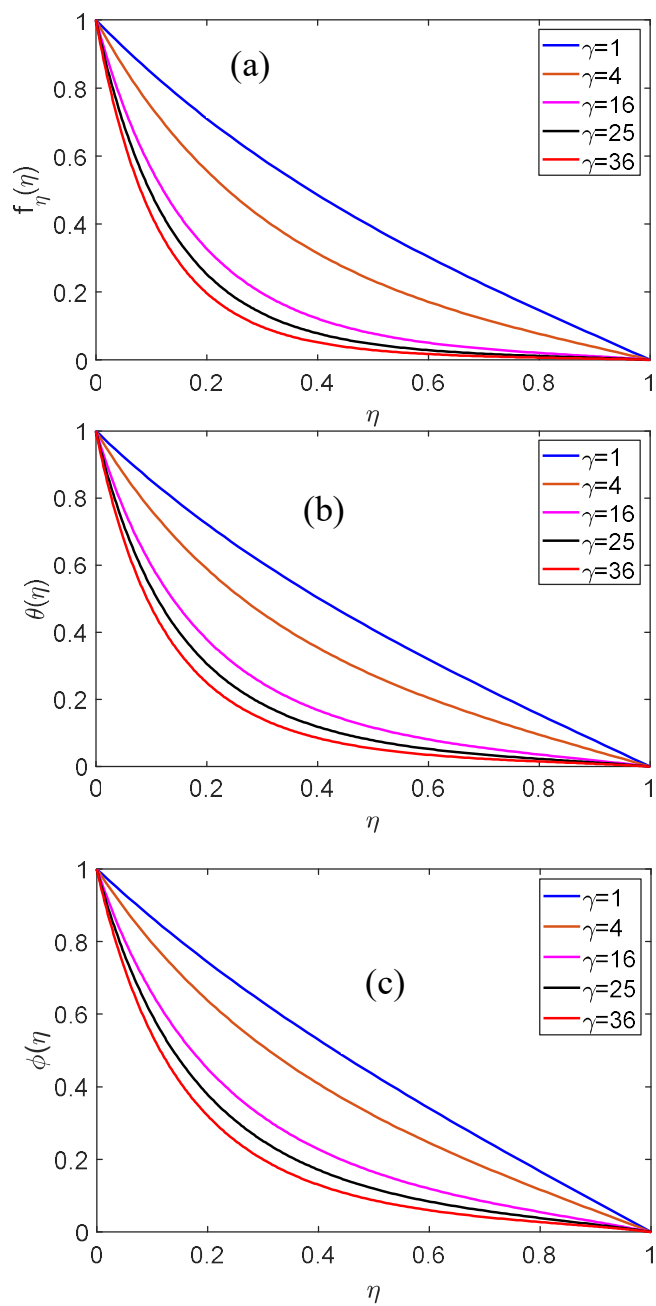

Figure 2(a-c): Velocity, temperature and concentration distributions with $\eta$ varying $\gamma$ when $D a=0.6, \operatorname{Pr}=0.71, S c=0.5$ and $S=0.8$
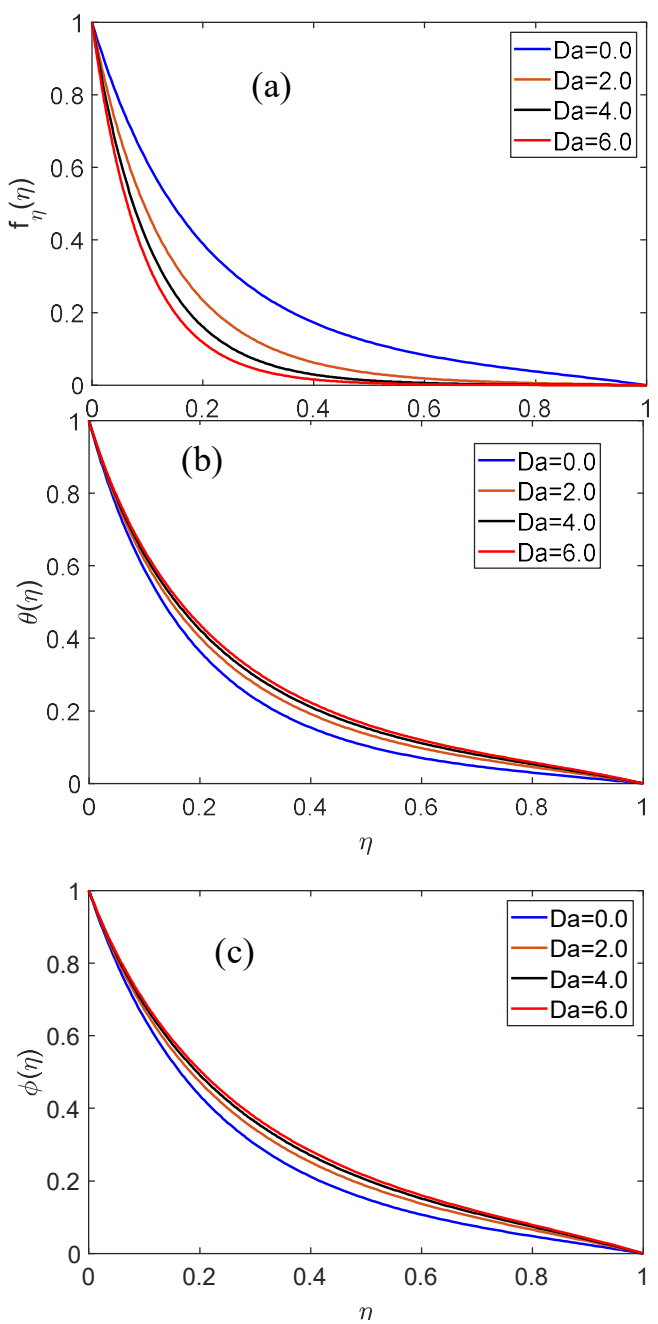

Figure 3(a-c): Velocity, temperature and concentration distributions with $\eta$ varying $D a$ when $\gamma=16, \operatorname{Pr}=0.71, S c=0.5$ and $S=0.8$

The influence of the unsteadiness parameter $S$ on the velocity, temperature as well as concentration distributions are shown in Figure 4(a-c). From figure 4(a) it has been observed that with an increase in unsteadiness parameters $S$ the velocity decreases along the surface. Also, with an increase in unsteadiness parameters $S$ the same behavior for the temperature as well as concentration profiles are observed in Figs. 4(b) as well as 4(c), respectively.

The temperature distribution for varying values of Prandtl number $\left(P_{r}\right)$ against $\eta$ has been displayed in figure 5 . From the graph it is observed that the temperature reduces with the increasing value of $P_{r}$ for a fixed value of $\eta$. Since a higher Prandtl number has comparatively low thermal conductivity, it lessens conduction of heat and therefore, temperature diminishes. Hence the heat transfer rate increases due to the increase in magnitude of $P_{r}$ so that the temperature distributions reduce.

The impacts of Schmidt number $(S c)$ on the species concentration distributions are observed in figure 6. From this figure it is noticed that an increase of $S c$ leads to thinning concentration boundary layer and hence the concentration profiles decrease. As the higher values of $S c$ representing heavier species, increase of $S c$ lower down the concentration level in the boundary layer. This is happened due to the fact that the Schmidt number $(S c)$ increases the molecular diffusivity of the chemical species decreases.

The variation of local skin-friction $C_{f}$ against local Reynolds number $R e_{x}$ with varying the Darcy number $D a$ has been displayed in Figure 7. The local skin-friction increases for increasing the values of the Darcy number along the local Reynolds number. Figure 8 represents Variation of local Nusselt number distribution $N u_{x}$ against Local Reynolds number $R e_{x}$ with varying the Prandtl number $P_{r}$. We see from figure 8 that the local Nusselt number 
$N u_{x}$ which is proportional to the rate of heat transfer $\theta^{\prime}(0)$ is increased with the increase of the Prandtl number $P_{r}$ against the local Reynolds number $R e_{x}$. Figure 9 demonstrates the variation of local Sherwood number $S h_{x}$ against local Reynolds number $R e_{x}$ with varying the Schmidt number $S c$. From figure 9 we see that the local Sherwood number $S h_{x}$ which is proportional to the rate of mass transfer $\phi^{\prime}(0)$ is increased with the increase of the Schmidt number $S c$ against the local Reynolds number $R e_{x}$. These phenomena are more clearly observed in Table 1 to Table 3, respectively.

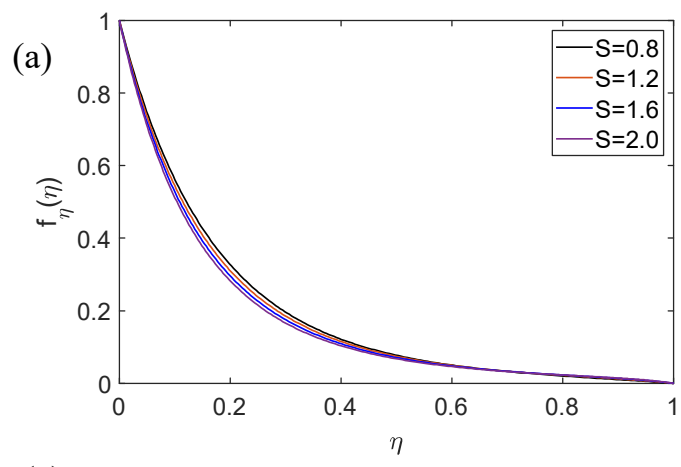

(c)
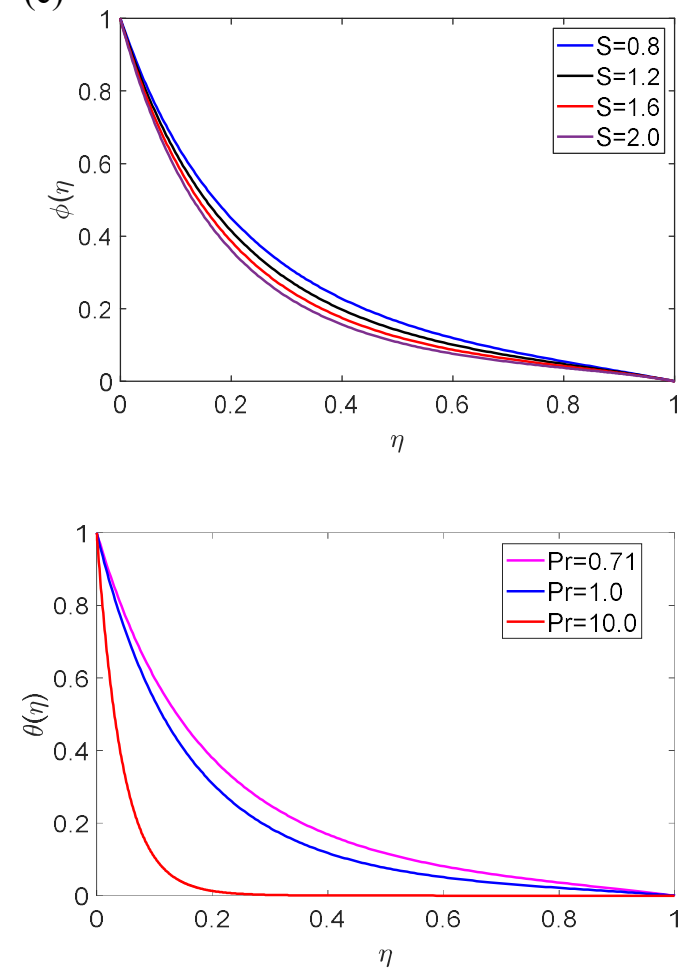

Figure 5: Temperature distribution with $\eta$ varying $P_{r}$ when $D a=0.6, S c=0.5, S=0.8$ and $\gamma=16$ (b)

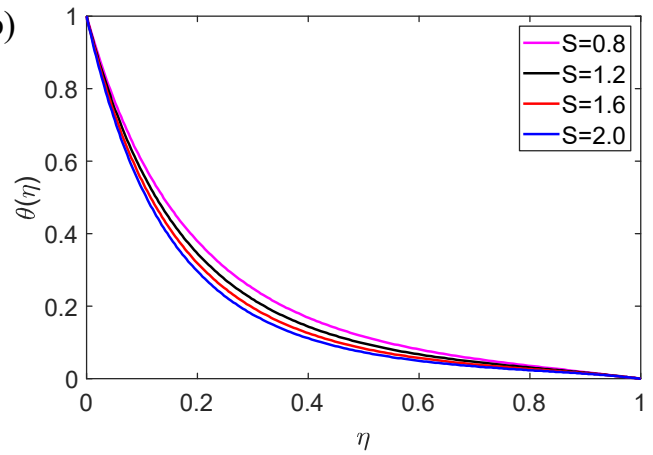

Figure 4(a-c): Velocity, temperature and concentration distributions with $\eta$ varying $S$ when $D a=0.6, \operatorname{Pr}=0.71, S c=0.5$ and $\gamma=16$

Table 1: Computed values for the local skin-friction, heat transfer rate and concentration rate with the variation of Darcy parameter $D a$ when $\gamma=\beta^{2}=16, S=0.8, P_{r}=0.71$ and $S c=0.5$.

\begin{tabular}{cccc}
\hline$D a$ & $f_{\eta \eta}(0)$ & $-\theta_{\eta}(0)$ & $-\phi_{\eta}(0)$ \\
\hline 0.0 & -0.2182 & 0.1619 & 0.2516 \\
0.6 & -0.1170 & 0.2021 & 0.2972 \\
1.2 & -0.0649 & 0.2341 & 0.3321 \\
1.8 & -0.0373 & 0.2601 & 0.3594 \\
\hline
\end{tabular}


Table 2: Computed values for the local skin-friction, heat transfer rate and concentration rate with the variation of Prandtl number $P_{r}$ when $\gamma=\beta^{2}=16, S=0.8, D a=0.6$ and $S c=0.5$.

\begin{tabular}{cccc}
\hline$P_{r}$ & $f_{\eta \eta}(0)$ & $-\theta_{\eta}(0)$ & $-\phi_{\eta}(0)$ \\
\hline 0.71 & -0.1170 & 0.2021 & 0.2972 \\
1.0 & -0.1170 & 0.1264 & 0.2972 \\
10.0 & -0.1170 & 0.0001 & 0.2972 \\
\hline
\end{tabular}

Table 3: Computed values for the local skin-friction, heat transfer rate and concentration rate with the variation of Schmidt number $S c$ when $\gamma=\beta^{2}=16, S=0.8, D a=0.6$ and $P_{r}=0.71$.

\begin{tabular}{|c|c|c|c|}
\hline & $f_{\eta \eta}(0)$ & $-\theta_{\eta}(0)$ & $\left(-\phi_{\eta}(0)\right)$ \\
\hline 0.5 & -0.1170 & -0.2021 & 0.2972 \\
\hline 1.0 & -0.1170 & -0.2021 & 0.1264 \\
\hline 1.5 & -0.1170 & -0.2021 & 0.0632 \\
\hline 2.0 & -0.1170 & -0.2021 & 0.0350 \\
\hline
\end{tabular}

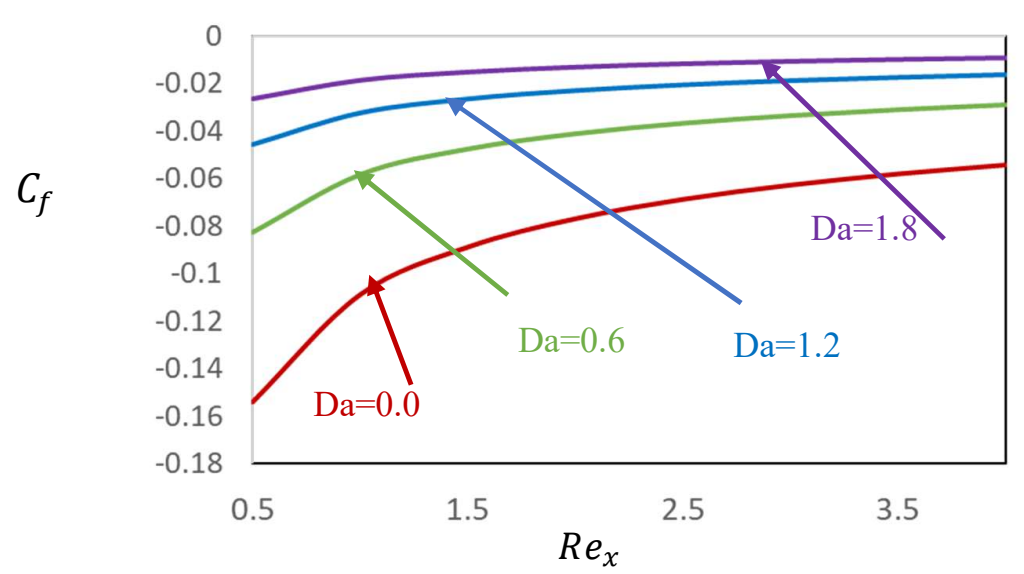

Figure 7: Variation of skin-friction $C_{f}$ against Reynolds number $R e_{x}$ with varying $D a$

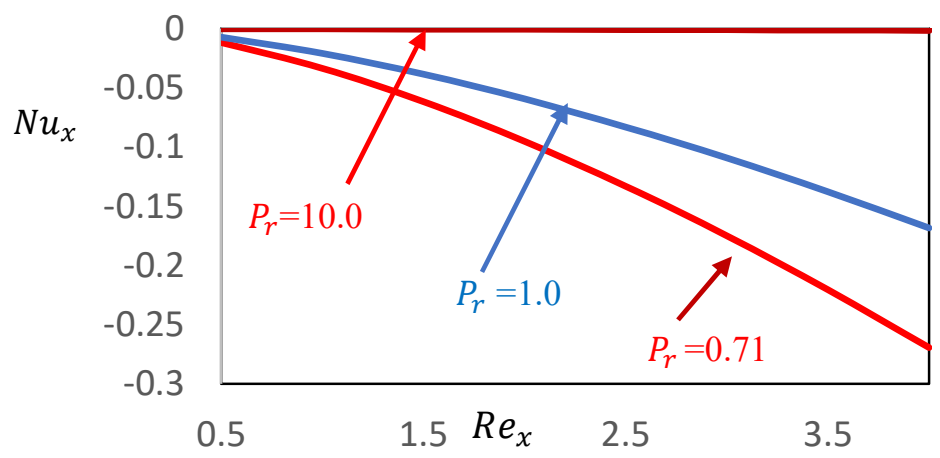

Figure 8: Variation of local Nusselt number distribution $N u_{x}$ against Reynolds number $R e_{x}$ with varying $P_{r}$ 


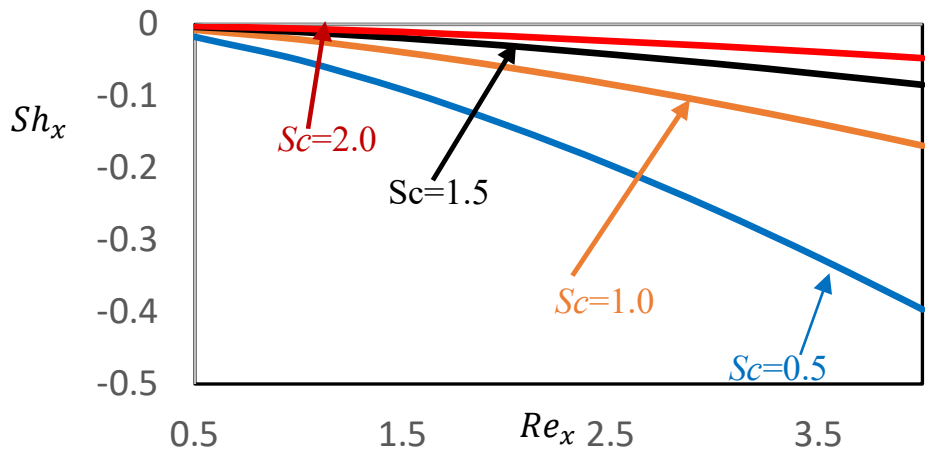

Figure 9: Variation of local Sherwood number $S h_{x}$ against Reynolds number $R e_{x}$ with varying $S c$

\section{CONCLUSIONS}

An investigation for unsteady convective heat and mass transfer in a thin liquid film over moving sheet in a saturated permeable surface has been carried out to analyze the impacts of permeability, thermal diffusivity and mass diffusion. The impacts of different parameters or numbers on the velocity, temperature and concentration profiles have been investigated through the research very carefully.

Important observations regarding the effects of the parameters/numbers on different profiles are as follows:

- With an increase in Darcy number the velocity decrease but opposite behavior for the temperature, the concentration and the local skin-friction increase.

- The rate of heat transfer increases for increasing the magnitude of Prandtl number so that the temperature distributions reduce.

- The higher values of Schmidt number representing heavier species, increase of Schmidt number lower down the concentration level in the boundary layer.

\section{REFERENCES}

Acrivos A., 1960. A Theoretical Analysis of Laminar Natural Convection Heat Transfer to Non-Newtonian Fluids, AIChE J, 6(4), 584-590.

Chen T. V. W., and Wollersheim D. E., 1973. Free Convection at a Vertical Plate with Uniform Flux Conditions in Non-Newtonian Power-Law Fluids, ASME J. Heat Transfer, 95, 123-124.

Chen, H.T., Chen, C.K., 1988. Natural convection of non-Newtonian fluids along a vertical plate embedded in a porous medium, ASME J. Heat Transfer, 110, 257-260.

Cheng, P., 1977. Combined free and forced convection flow about inclined surfaces in porous media, Int. J. Heat Mass Transfer 20, 807-814.

Degan G., Akowanou C., Awanou N. C., 2007. Transient natural convection of non-Newtonian fluids about a vertical surface embedded in an anisotropic porous medium, International Journal of Heat and Mass Transfer, 50(23), 4629-4639.

Emery A. F., Chi H. S., and Dale J. D., 1970. Free Convection Through Vertical Plane Layers of Non-Newtonian Power-Law Fluids, ASME J. Heat Transfer, 93(2), 164-171.

Hooper, W.B., Chen, T.S., Armaly, B.F., 1993. Mixed convection from a vertical plate in porous media with surface injection or suction, Numer. Heat Transfer 25, 317-329.

Huang, M. J., Huang, J. S., Chou, Y. L., and Cheng, C. K., 1989. Effects of Prandtl Number on Free Convection Heat Transfer from a Vertical Plate to a Non-Newtonian Fluid, ASME J. Heat Transfer, 111(1), 189-91.

Khader M. M., Megahed Ahmed M., 2013. Numerical simulation using the finite difference method for the flow and heat transfer in a thin liquid film over an unsteady stretching sheet in a saturated porous medium in the presence of thermal radiation, Journal of King Saud University - Engineering Sciences, 25, 29-34.

Merkin, J.H., 1985. On dual solutions occurring in mixed convection in a porous medium, J. Eng. Math., 20, 171179.

Molla M. M., and Yao L. S., 2009. Non-Newtonian natural convection along a vertical heated wavy surface using a modified power-law viscosity mode, J. Heat Transfer, 131(1): 012501-012507.

Nield, D.A., Bejan, A., 1984. Convection in Porous Media, Springer, New York.

Noor, N.F.M., Hashim, I., 2010. Thermocapillary and magnetic field effects in a thin liquid film on an unsteady stretching surface, Int. J. Heat Mass Transfer, 53, 2044-2051.

Rashad A. M., EL-Hakiem M. A., Abdou M. M. M., 2011. Natural convection boundary layer of a non-Newtonian fluid about a permeable vertical cone embedded in a porous medium saturated with a nanofluid, Computers \& Mathematics with Applications, 62(8), 3140-3151. 
Rashad, A., Chamkha, Ali J., Abdou, M. M. M., 2013. Mixed Convection Flow of Non-Newtonian Fluid from Vertical Surface Saturated in a Porous Medium Filled with a Nanofluid, Journal of Applied Fluid Mechanics, 6 (2), 301-309.

Shenoy A. V., 1994. Non-Newtonian fluid heat transfer in porous media, Advances in Heat Transfer, 24, 101190.

Shenoy, A.V., 1993. Darcy-Forchheimer natural, forced and mixed convection heat transfer in non-Newtonian power-law fluid saturated porous media, Trans. Porous Med., 11, 219-241.

Wright, S.D., Ingham, D.B., Pop, I., 1996. On natural convection from a vertical plate with a prescribed surface heat flux in porous media, Trans. Porous Med. 22, 181-193.

Yih, K.A., 1997. The effect of uniform lateral mass flux on free convection about vertical cone embedded in a saturated porous medium, Int. Commun. Heat Mass Transfer 24, 1195-1205.

(C) 2021 the Authors. Journal of Engineering Science published by Faculty of Civil Engineering, Khulna University of Engineering \& Technology. This is an open access article under the terms of the Creative Commons AttributionNonCommercial-NoDerivatives License, which permits use and distribution in any medium, provided the original work is properly cited, the use is non-commercial and no Modifications or adaptations are made. 